\title{
Approximate Spacetime for Neutron Stars
}

\author{
Francisco Frutos-Alfaro \\ School of Physics, Space Research Center \\ and \\ Laboratory of Theoretical Physics and Computation of the University of Costa Rica
}

September 4, 2019

\begin{abstract}
An approximate realistic metric representing the spacetime of neutron stars is obtained by perturbing the Kerr metric. This metric has five parameters, namely the mass, spin or angular momentum, mass quadrupole, spin octupole and mass hexadecapole. Moreover, a version of the Hartle-Thorne metric containing these parameters is constructed by means of a series transformation between these spacetimes and solving the Einstein field equations. The form of the Pappas metric in Schwarzschild spherical coordinates is found. The three relativistic multipole structures are compared.
\end{abstract}

\section{Introduction}

Among compact objects are neutron stars (NS). These stars are relativistic rotating objects with high density, and strong gravitational and magnetic fields. The study of NS is relevant to understand the extrem conditions of matter in there, the behaviour of particles around them, and the structure of its spacetime [12, 3, 1].

The quest to find a realistic spacetime representation for neutron stars (NS) is an important task in astrophysics. Many attemps to obtain this spacetime have been done from approximate metrics until numerical metrics. The first attemp was made by Hartle-Thorne (HT). The relevance of the HT work was that they matched the interior solution with the exterior one [11]. Quevedo and Mashhoon [18] and Manko and Novikov 14 obtained exact solutions with charge and arbitrary mass multipoles. Later, more exact solutions containing other features, for instance magnetic dipole, were found [15]. With the advent of computer technology, the implementations of computer programs to find numerical solutions become a vogue [20. However, approximate solutions are still important to extract astrophysical information from NS [17]. Moreover, a fourth order HT metric for the exterior of neutron stars was obtained in [21].

There are several techniques to find solutions of the Einstein field equations (EFE). Among them, the Ernst formalism has played an important role in finding new exact and approximate solutions. This formalism is employed in [18, 14, 17]. In this contribution, however, we include features like mass quadrupole, spin octupole and mass hexadecapole to the Kerr metric perturbatively. This is achieved easily by means of perturbing the Lewis metric form of the Kerr spacetime [7, 8]. The form of the perturbations due to spin octupole and mass hexadecapole has the structure proposed by Ryan [19]. Then, one is certain that these features are introduced in the right manner. This metric has the advantage that it reduces to the Kerr metric which is an exact solution with mass and angular momentum. Moreover, it is simple to implement computationaly. 
The HT spacetime does not have spin octupole, mass hexadecapole, and the interactions massquadrupole, quadrupole-quadrupole, and spin-quadrupole. Nevertheless, this metric gives excelent results of the inner most stable circular orbit (ISCO) of particles around NS [2]. Adding these interactions, and the spin octupole and the mass hexadecapole to this metric would improve considerably its applicability in computational calculations. One can guess an approximate HT metric with these features from our deformed Kerr spacetime, by finding a transformation between them from the post-linear forms of these metrics and solving the EFE. Both spacetime were tested to be solutions of the EFE by means of REDUCE programs, and these programs are available upon request.

This paper is organized as follows. The perturbation method of the Kerr metric using the Lewis spacetime is discussed in section 2. In Section 3, the construction of the HT version is presented via a series transformation for the first order in spin octupole and mass hexadecapole. The inclusion of the interactions of the spin octupole and mass hexadecapole with the mass, spin, quadrupole and with each other is also found in this section. The relativistic multipole structure is found using the Fodor-Hoenselaers-Perjés method [6] in Section 4. In Section 5, the Pappas metric [17] is compared with the ones presented here. In the last section some conclusions are presented.

\section{Generating the Metric}

The Lewis metric [13] was successfully applied to find approximate solutions of the EFE using the Erez-Rosen metric and the Kerr as seed metrics [7, 8]. It is given by

$$
d s^{2}=-V d t^{2}+2 W d t d \phi+X d \rho^{2}+Y d z^{2}+Z d \phi^{2}
$$

where the chosen canonical coordinates are $x^{1}=\rho$ and $x^{2}=z$. The potentials $V, W, X, Y$ and $Z$ are functions of $\rho$ and $z$ with $\rho^{2}=V Z+W^{2}$.

The transformation that leads to the Kerr metric is

$$
\rho=\sqrt{\Delta} \sin \theta \quad \text { and } \quad z=(r-m) \cos \theta,
$$

where $\Delta=r^{2}-2 m r+a^{2}, m$ and $a$ are the mass and the rotational parameter. The angular momentum or spin is given by $\mathcal{S}_{1}=J=m a$.

The Ansatz for the Lewis potentials to include the spin octupole parameter, $S_{3}$ and the mass hexadecapole parameter, $M_{4}$, is

$$
\begin{aligned}
V & =V_{K} \mathrm{e}^{-2 \psi} \\
W & =W_{K}+W_{m s}, \\
X & =\left(X_{K}+X_{m s}\right) \mathrm{e}^{2 \chi} \\
Y & =\left(Y_{K}+Y_{m s}\right) \mathrm{e}^{2 \chi} \\
Z & =\left(Z_{K}+Z_{m s}\right) \mathrm{e}^{2 \psi}
\end{aligned}
$$

where the potentials $V_{K}, W_{K}, X_{K}, Y_{K}, Z_{K}$ are the Lewis potentials for the Kerr metric. The perturbation terms are $W_{m s}, X_{m s}, Y_{m s}, Z_{m s}$ which include terms with $M_{4}$ and $S_{3}$, and interaction of these parameters with the other ones.

The functions $\psi, \chi, W_{m s}, X_{m s}, Y_{m s}$, and $Z_{m s}$ are chosen as follows 


$$
\begin{aligned}
\psi & =\psi_{q}+\psi_{m s}, \\
\chi & =\chi_{q}+\chi_{m s}, \\
W_{m s} & =\left(\xi_{0} \frac{J q}{r^{4}}+\xi_{1} \frac{S_{3}}{r^{3}}\right) h_{31}+\xi_{2} \frac{m S_{3}}{r^{4}} h_{32}+\xi_{3} \frac{q S_{3}}{r^{6}} h_{33}+\xi_{4} \frac{M_{4} S_{3}}{r^{8}} h_{34}+\xi_{5} \frac{J M_{4}}{r^{6}} h_{35}, \\
X_{m s} & =\mu_{1} \frac{m M_{4}}{r^{6}} h_{41}+\mu_{2} \frac{q M_{4}}{r^{8}} h_{42}+\mu_{3} \frac{M_{4}^{2}}{r^{10}} h_{43}, \\
Y_{m s} & =\iota_{1} \frac{m M_{4}}{r^{4}} h_{51}+\iota_{2} \frac{q M_{4}}{r^{6}} h_{52}+\iota_{3} \frac{M_{4}^{2}}{r^{8}} h_{53}+\iota_{4} \frac{S_{3}^{2}}{r^{6}} h_{54},+\iota_{5} \frac{J S_{3}}{r^{4}} h_{55}, \\
Z_{m s} & =\left(\zeta_{1} \frac{m M_{4}}{r^{4}} h_{51}+\zeta_{2} \frac{q M_{4}}{r^{6}} h_{52}+\zeta_{3} \frac{M_{4}^{2}}{r^{8}} h_{53}+\zeta_{4} \frac{S_{3}^{2}}{r^{6}} h_{54}+\zeta_{5} \frac{J S_{3}}{r^{4}} h_{55}\right) \sin ^{2} \theta,
\end{aligned}
$$

where

$$
\begin{aligned}
\psi_{q} & =\frac{q}{r^{3}} P_{2}+3 \frac{m q}{r^{4}} P_{2}, \\
\chi_{q} & =\frac{q P_{2}}{r^{3}}+\frac{1}{3} \frac{m q}{r^{4}}\left(5 P_{2}^{2}+5 P_{2}-1\right)+\frac{1}{9} \frac{q^{2}}{r^{6}}\left(25 P_{2}^{3}-21 P_{2}^{2}-6 P_{2}+2\right), \\
\psi_{m s} & =\gamma_{1} \frac{M_{4}}{r^{5}} h_{11}+\gamma_{2} \frac{m M_{4}}{r^{6}} h_{12}+\gamma_{3} \frac{q M_{4}}{r^{8}} h_{13}+\gamma_{4} \frac{S_{3}^{2}}{r^{8}} h_{14}+\gamma_{5} \frac{J S_{3}}{r^{6}} h_{15}, \\
\chi_{m s} & =\eta_{1} \frac{M_{4}}{r^{5}} h_{21}+\eta_{2} \frac{m M_{4}}{r^{6}} h_{22}+\eta_{3} \frac{q M_{4}}{r^{8}} h_{23}+\eta_{4} \frac{M_{4}^{2}}{r^{10}} h_{24}+\eta_{5} \frac{S_{3}^{2}}{r^{8}} h_{25}+\eta_{6} \frac{J S_{3}}{r^{6}} h_{26} .
\end{aligned}
$$

In [10], $\psi_{q}$ and $\chi_{q}$ were found. The functions $h_{i j}$ are functions of $\theta$ only. The first order terms of $\psi_{m s}, \chi_{m s}$ and $W_{m s}$ are taken from [19]. The term $J q$ of $W_{m s}$ represents the interaction of the spin with the quadrupole, which was not considered in [10.

From this Ansatz, the perturbative terms can be determined by solving the EFE. The functions $h_{i j}$ are combinations of Legendre polynomials, $P_{n}(\cos \theta), n=1, \ldots, 8$, and an associated Legendre polynomial, $P_{3}^{1}(\cos \theta)=\left(5 P_{2}+1\right) \sin \theta$. After solving the EFE, the functions $h_{i j}$ are given by

$$
\begin{aligned}
& h_{11}=P_{4}, \\
& h_{12}=P_{4}, \\
& h_{13}=P_{7}, \\
& h_{14}=\frac{7}{64}+\frac{7}{48} P_{2}+\frac{63}{352} P_{4}+\frac{175}{528} P_{6}+\frac{16}{429} P_{7}, \\
& h_{15}=-\frac{3}{4} P_{2}-P_{4}, \\
& h_{21}=P_{4}, \\
& h_{22}=\frac{45}{11} P_{4}, \\
& h_{23}=P_{7}, \\
& h_{24}=-\left(\frac{5}{99}+\frac{625}{9009} P_{2}+\frac{90}{1001} P_{4}+\frac{250}{1683} P_{6}+\frac{12250}{24453} P_{8}\right), \\
& h_{25}=-\frac{287}{1728}-\frac{91}{528} P_{2}-\frac{7}{416} P_{4}+\frac{175}{144} P_{6}+\frac{16}{429} P_{7}, \\
& h_{26}=\frac{5}{4} P_{2}-3 P_{4}, \\
& h_{31}=P_{3}^{1} \sin \theta,
\end{aligned}
$$




$$
\begin{aligned}
& h_{32}=\frac{5}{2} P_{2}-\frac{8}{63} P_{3}-\frac{5}{2} P_{4}+\frac{8}{63} P_{5}, \\
& h_{33}=-\frac{1}{10}-\frac{1}{6} P_{2}-\frac{27}{55} P_{4}+\frac{25}{33} P_{6}, \\
& h_{34}=-\frac{5}{33} P_{2}-\frac{25}{143} P_{4}-\frac{35}{66} P_{6}+\frac{245}{286} P_{8}, \\
& h_{35}=\frac{20}{11}\left(P_{4}-P_{6}\right) \\
& h_{41}=P_{3}, \\
& h_{42}=-\frac{24}{7} P_{2}+\frac{600}{77} P_{4}+\frac{300}{143} P_{6}, \\
& h_{43}=P_{4}, \\
& h_{51}=\frac{2}{33}-\frac{2}{5} P_{1}+\frac{10}{33} P_{2}+\frac{2}{5} P_{3}+\frac{6}{11} P_{4}, \\
& h_{52}=-\frac{3}{26}+\frac{47}{26} P_{2} P_{4}-\frac{303}{182} P_{2}-\frac{5}{182} P_{4}, \\
& h_{53}=\frac{33191}{4157010}+\frac{33191}{831402} P_{2}+\frac{165259}{692835} P_{4}+\frac{882}{3553} P_{6}+\frac{882}{2717} P_{8}, \\
& h_{54}=\frac{125293}{247104}+\frac{35315}{82368} P_{2}-\frac{581}{9152} P_{4}-\frac{6125}{6336} P_{6}, \\
& h_{55}=3\left(P_{4}-P_{2}\right) .
\end{aligned}
$$

The constants are found to be

$$
\begin{aligned}
\gamma_{1} & =1, \quad \gamma_{2}=5, \quad \gamma_{3}=\gamma_{4}=\gamma_{5}=1, \\
\eta_{1} & =\eta_{2}=\eta_{3}=\eta_{4}=\eta_{5}=\eta_{6}=1, \\
\xi_{0} & =1, \quad \xi_{1}=\frac{7}{12}, \quad \xi_{2}=\xi_{3}=\xi_{4}=\xi_{5}=1, \\
\mu_{1} & =4, \quad \mu_{2}=\mu_{3}=1, \\
\iota_{1} & =\iota_{2}=\iota_{3}=\iota_{4}=\iota_{5}=1, \\
\zeta_{1} & =\zeta_{2}=\zeta_{3}=\zeta_{4}=\zeta_{5}=-1 .
\end{aligned}
$$

The new metric potentials are

$$
\begin{aligned}
V & =\frac{1}{\Sigma^{2}}\left[\Delta-a^{2} \sin ^{2} \theta\right] \mathrm{e}^{-2 \psi} \\
W & =-\frac{2 J r}{\Sigma^{2}} \sin ^{2} \theta+W_{m s} \\
X & =\left(\frac{\Sigma^{2}}{\Delta}+X_{m s}\right) \mathrm{e}^{2 \chi} \\
Y & =\left(\Sigma^{2}+Y_{m s}\right) \mathrm{e}^{2 \chi} \\
Z & =\left(\frac{1}{\Sigma^{2}}\left[\left(r^{2}+a^{2}\right)^{2}-a^{2} \Delta \sin ^{2} \theta\right]-Y_{m s}\right) \mathrm{e}^{2 \psi} \sin ^{2} \theta
\end{aligned}
$$

where $\Sigma^{2}=r^{2}+a^{2} \cos ^{2} \theta$.

This metric is valid up to third order in all parameters, including the interactions of all parameters with each other.

A post-linear expansion of the metric can be written as 


$$
\begin{aligned}
V & \simeq\left(1-2 U-2 \frac{m a^{2}}{r^{3}} \cos ^{2} \theta\right) \mathrm{e}^{-2 \psi} \\
W & \simeq-2 \frac{J}{r} \sin ^{2} \theta+W_{m s} \\
X & \simeq\left(1+2 U+4 U^{2}-\frac{a^{2}}{r^{2}} \sin ^{2} \theta-2 \frac{m a^{2}}{r^{3}}\left(1+\sin ^{2} \theta\right)-4 \frac{m^{2} a^{2}}{r^{4}}\left(2+\sin ^{2} \theta\right)+X_{m s}\right) \mathrm{e}^{2 \chi} \\
Y & =r^{2}\left(1+\frac{a^{2}}{r^{2}} \cos ^{2} \theta+\frac{Y_{m s}}{r^{2}}\right) \mathrm{e}^{2 \chi}, \\
Z & \simeq r^{2} \sin ^{2} \theta\left(1+\frac{a^{2}}{r^{2}}+2 \frac{m a^{2}}{r^{3}} \sin ^{2} \theta-\frac{Y_{m s}}{r^{2}}\right) \mathrm{e}^{2 \psi},
\end{aligned}
$$

where $U=m / r$. In [10] is the complete expansion without the $S_{3}$ and $M_{4}$ terms.

\section{Constructing a new Hartle-Thorne Metric}

Adding perturbatively some features, for example the spin octupole and the mass hexadecapole to the HT metric would be interesting, because this metric is still used as a comparison with more realistic metrics. The HT metric is an approximate solution of the EFE with three parameters, mass, angular momentum and mass quadrupole. It is given by

$$
\begin{aligned}
d s^{2} & =-F_{1} d t^{2}+F_{2} d R^{2}+R^{2} F_{3}\left[d \theta^{2}+\sin ^{2} \theta(d \phi-\omega d t)^{2}\right] \\
& =-V_{H T} d t^{2}-2 W_{H T} d t d \phi+X_{H T} d R^{2}+R^{2} Y_{H T}\left[d \theta^{2}+\sin ^{2} \theta d \phi^{2}\right]
\end{aligned}
$$

where

$$
\begin{aligned}
F_{1} & =\left(1-2 U+2 \frac{J^{2}}{R^{4}}\right)\left[1+2 K_{1} P_{2}\right] \\
& \simeq\left(1-2 U+2 \frac{J^{2}}{R^{4}}\right) \mathrm{e}^{2 \psi_{1}}, \\
F_{2} & =\left[1-2 K_{2} P_{2}\right]\left(1-2 U+2 \frac{J^{2}}{R^{4}}\right)^{-1} \\
& \simeq \mathrm{e}^{-2 \psi_{2}}\left(1-2 U+2 \frac{J^{2}}{R^{4}}\right)^{-1}, \\
F_{3} & =1-2 K_{3} P_{2} \simeq \mathrm{e}^{-2 \psi_{3}}, \\
\omega & =2 \frac{J}{R^{3}} .
\end{aligned}
$$

The functions $K_{1}, K_{2}$, and $K_{3}$ are given by

$$
\begin{aligned}
& K_{1}=\frac{J^{2}}{m R^{3}}(1+U)+\frac{5}{8}\left(\frac{q}{m^{3}}-\frac{J^{2}}{m^{4}}\right) Q_{2}^{2}\left(\frac{R}{m}-1\right), \\
& K_{2}=K_{1}-6 \frac{J^{2}}{R^{4}}=K_{1}-6 \frac{J^{2}}{m^{4}} U^{4}
\end{aligned}
$$




$$
K_{3}=\left(K_{1}+\frac{J^{2}}{R^{4}}\right)+\frac{5}{4}\left(\frac{q}{m^{3}}-\frac{J^{2}}{m^{4}}\right) \frac{U}{\sqrt{1-2 U}} Q_{2}^{1}\left(\frac{R}{m}-1\right),
$$

where $U=M / R$. The functions $Q_{2}^{1}$ and $Q_{2}^{2}$ are Legendre functions of the second kind

$$
\begin{aligned}
& Q_{2}^{1}(x)=\sqrt{x^{2}-1}\left(\frac{3}{2} x \ln \left(\frac{x+1}{x-1}\right)-\frac{\left(3 x^{2}-2\right)}{\left(x^{2}-1\right)}\right) \\
& Q_{2}^{2}(x)=\left(x^{2}-1\right)\left(\frac{3}{2} \ln \left(\frac{x+1}{x-1}\right)-\frac{\left(3 x^{3}-5 x\right)}{\left(x^{2}-1\right)^{2}}\right) .
\end{aligned}
$$

The metric potencials are

$$
\begin{aligned}
V_{H T} & \simeq\left(1-2 U-\frac{2}{3} \frac{J^{2}}{R^{4}}\right) \mathrm{e}^{2 \alpha_{1}}, \\
W_{H T} & =-2 \frac{J}{R} \sin ^{2} \theta \\
X & \simeq\left(1-2 U+2 \frac{J^{2}}{R^{4}}\right)^{-1} \mathrm{e}^{-2 \alpha_{2}}, \\
Y_{H T} & \simeq \mathrm{e}^{-2 \alpha_{3}},
\end{aligned}
$$

where

$$
\begin{aligned}
\alpha_{1} & =\left(K_{1}+\frac{4}{3} \frac{J^{2}}{R^{4}}\right) P_{2}, \\
\alpha_{2} & =K_{2} P_{2}, \\
\alpha_{3} & =K_{3} P_{2} .
\end{aligned}
$$

The Taylor expansion of $K_{1}, K_{2}$ and $K_{3}$ are

$$
\begin{aligned}
& K_{1}=\frac{q}{R^{3}}+3 \frac{m q}{R^{4}}-2 \frac{J^{2}}{R^{4}}, \\
& K_{2}=\frac{q}{R^{3}}+3 \frac{m q}{R^{4}}-8 \frac{J^{2}}{R^{4}}, \\
& K_{3}=\frac{q}{R^{3}}+\frac{5}{2} \frac{m q}{R^{4}}-\frac{1}{2} \frac{J^{2}}{R^{4}} .
\end{aligned}
$$

The complete expansion of the HT including the second order terms in $q$ was found in [9, 10].

To guess an improvement of the HT metric, we have to find a solution of the EFE compatible with HT metric. In order to do it, we will propose an Ansatz. In [9], the second order in $q$ for the post-linear HT was found perturbatively. A transformation that converts the post-linear Kerr-like metric (7) without $S_{3}$ and $M_{4}$ into an improved HT was obtained in [10]. The same transformation can be used to transform the post-linear Kerr-like metric (17) with $S_{3}$ and $M_{4}$ at first order into an improved HT in the post-linear form of (11) with $S_{3}$ and $M_{4}$ at first order, changing $q \rightarrow m a^{2}-q$. This transformation is 10 .

$$
\begin{aligned}
& r=R\left[1+\frac{m q}{R^{4}} f_{1}+\frac{q^{2}}{R^{6}} f_{2}+\frac{a^{2}}{R^{2}}\left(h_{1}+\frac{m}{R} h_{2}+\frac{m^{2}}{R^{2}} h_{3}\right)\right] \\
& \theta=\Theta+\frac{m q}{R^{4}} g_{1}+\frac{q^{2}}{R^{6}} g_{2}+\frac{a^{2}}{R^{2}}\left(h_{4}+\frac{m}{R} h_{5}\right)
\end{aligned}
$$


where

$$
\begin{aligned}
f_{1} & =\frac{1}{9}\left(5 P_{2}^{2}-4 P_{2}-1\right), \\
f_{2} & =\frac{1}{72}\left(40 P_{2}^{3}-24 P_{2}^{2}-43\right), \\
g_{1} & =\frac{1}{6}\left(2-5 P_{2}\right) \cos \Theta \sin \Theta, \\
g_{2} & =\frac{1}{6} P_{2}\left(2-5 P_{2}\right) \cos \Theta \sin \Theta, \\
h_{1} & =-\frac{1}{2} \sin ^{2} \Theta, \\
h_{2} & =-\frac{1}{2} \sin ^{2} \Theta, \\
h_{3} & =1-3 \cos ^{2} \Theta=-2 P_{2}, \\
h_{4} & =-\frac{1}{2} \cos \Theta \sin \Theta, \\
h_{5} & =-\cos \Theta \sin \Theta,
\end{aligned}
$$

with $P_{2}=P_{2}(\cos \Theta)$

Now, considering this fact, the $J q$ interaction term and the terms due to the spin octupole $S_{3}$ and the mass hexadecapole $M_{4}$ at second order, the Ansatz of an improved HT metric functions is from (7)

$$
\begin{aligned}
V & \simeq\left(1-2 U-\frac{2}{3} \frac{J^{2}}{R^{4}}\right) \mathrm{e}^{2 \psi_{1}} \\
W & \simeq-\left[2 \frac{J}{R}+\left(\frac{J q}{R^{4}}-\frac{7}{12} \frac{S_{3}}{R^{3}}\right)\left(5 P_{2}+1\right)\right] \sin ^{2} \Theta+\tilde{W}_{m s} \\
X & \simeq\left(1-2 U+2 \frac{J^{2}}{R^{4}}\right)^{-1} \mathrm{e}^{-2 \psi_{2}} \\
Y & \simeq R^{2} \mathrm{e}^{-2 \psi_{3}} \\
Z & \simeq R^{2} \mathrm{e}^{-2 \psi_{3}} \sin ^{2} \Theta
\end{aligned}
$$

where

$$
\begin{aligned}
& \psi_{1}=\frac{q}{R^{3}} P_{2}+3 \frac{m q}{R^{4}} P_{2}-\frac{2}{3} \frac{J^{2}}{R^{4}} P_{2}+\frac{M_{4}}{R^{5}} P_{4}+\psi_{1 m s} \\
& \psi_{2}=\frac{q}{R^{3}} P_{2}+3 \frac{m q}{R^{4}} P_{2}+\frac{1}{24} \frac{q^{2}}{R^{6}}\left[16 P_{2}^{2}+16 P_{2}-77\right]-8 \frac{J^{2}}{R^{4}} P_{2}+\frac{M_{4}}{R^{5}} P_{4}+\psi_{2 m s} \\
& \psi_{3}=\frac{q}{R^{3}} P_{2}+\frac{5}{2} \frac{m q}{R^{4}} P_{2}+\frac{1}{72} \frac{q^{2}}{R^{6}}\left[28 P_{2}^{2}-8 P_{2}+43\right]-\frac{1}{2} \frac{J^{2}}{R^{4}} P_{2}+\frac{M_{4}}{R^{5}} P_{4}+\psi_{3 m s}
\end{aligned}
$$

with

$$
\begin{aligned}
& \psi_{1 m s}=\frac{m M_{4}}{r^{6}} \tilde{h}_{12}+\frac{q M_{4}}{r^{8}} \tilde{h}_{13}+\frac{J S_{3}}{r^{6}} \tilde{h}_{14}+\frac{S_{3}^{2}}{r^{8}} \tilde{h}_{15}, \\
& \psi_{2 m s}=\frac{m M_{4}}{r^{6}} \tilde{h}_{22}+\frac{q M_{4}}{r^{8}} \tilde{h}_{23}+\frac{J S_{3}}{r^{6}} \tilde{h}_{24}+\frac{M_{4}^{2}}{r^{10}} \tilde{h}_{25}+\frac{S_{3}^{2}}{r^{8}} \tilde{h}_{26},
\end{aligned}
$$




$$
\begin{aligned}
& \psi_{3 m s}=\frac{m M_{4}}{r^{6}} \tilde{h}_{32}+\frac{q M_{4}}{r^{8}} \tilde{h}_{33}+\frac{J S_{3}}{r^{6}} \tilde{h}_{34}+\frac{M_{4}^{2}}{r^{10}} \tilde{h}_{35}+\frac{S_{3}^{2}}{r^{8}} \tilde{h}_{36}, \\
& \tilde{W}_{m s}=\frac{m S_{3}}{r^{4}} \tilde{h}_{42}+\frac{q S_{3}}{r^{6}} \tilde{h}_{43}+\frac{M_{4} S_{3}}{r^{8}} \tilde{h}_{44}+\frac{J S_{3}}{r^{5}} \tilde{h}_{45}+\frac{J M_{4}}{r^{6}} \tilde{h}_{46} .
\end{aligned}
$$

The $\tilde{h}_{i j}$ are found solving the EFE perturbatively. These functions are

$$
\begin{aligned}
& \tilde{h}_{12}=3 P_{4}, \\
& \tilde{h}_{13}=P_{7} \text {, } \\
& \tilde{h}_{14}=\frac{3}{4} P_{2}+P_{4}, \\
& \tilde{h}_{15}=-\frac{7}{48} P_{2}-\frac{63}{352} P_{4}-\frac{175}{528} P_{6}-\frac{7}{64}, \\
& \tilde{h}_{22}=5 P_{4}-\frac{110}{27} \text {, } \\
& \tilde{h}_{23}=-\frac{20}{21} P_{2}+\frac{36}{77} P_{4}+\frac{16}{33} P_{6}+P_{7}-7, \\
& \tilde{h}_{24}=-\frac{15}{4} P_{2}+\frac{11}{2} P_{4}-5 \text {, } \\
& \tilde{h}_{25}=\frac{1000}{693} P_{2}+\frac{360}{1001} P_{4}+\frac{20}{99} P_{6}+\frac{280}{1287} P_{8}+\frac{5}{18}, \\
& \tilde{h}_{26}=-\frac{679}{144} P_{2}-\frac{1505}{1056} P_{4}-\frac{1085}{792} P_{6}-\frac{637}{96}, \\
& \tilde{h}_{32}=\frac{245}{27} P_{2}^{2}-\frac{70}{27} P_{2}-1 \text {, } \\
& \tilde{h}_{33}=\frac{5}{21} P_{2}+\frac{12}{77} P_{4}+\frac{47}{132} P_{6}+P_{7}+1 \text {, } \\
& \tilde{h}_{34}=\frac{7}{3} P_{4}+1 \text {, } \\
& \tilde{h}_{35}=-\frac{50}{693} P_{2}+\frac{45}{1001} P_{4}+\frac{8}{99} P_{6}+\frac{217}{1287} P_{8} \text {, } \\
& \tilde{h}_{36}=\frac{259}{576} P_{2}-\frac{35}{264} P_{4}-\frac{10255}{12672} P_{6}+1, \\
& \tilde{h}_{42}=\frac{5}{2}\left(P_{2}-P_{4}\right) \text {, } \\
& \tilde{h}_{43}=\frac{1}{6} P_{2}+\frac{27}{55} P_{4}-\frac{25}{33} P_{6}+\frac{1}{10}, \\
& \tilde{h}_{44}=\frac{5}{33} P_{2}+\frac{25}{143} P_{4}+\frac{35}{66} P_{6}-\frac{245}{286} P_{8}, \\
& \tilde{h}_{45}=0 \text {, } \\
& \tilde{h}_{46}=4 P_{2} P_{4}-\frac{8}{7} P_{2}-\frac{20}{7} P_{4} .
\end{aligned}
$$

This metric is solution of the EFE up to the third order in all parameters $\left(m, q, M_{4}, J, S_{3}\right)$.

\section{Relativistic Multipole Moments}

To determine if two metric are isometric, one has to compare its multipole structure. It is useful to find this structure for our spacetime. At first glance, our metric has 5 complex multipoles $\left(\mathcal{M}_{0}=m, \mathcal{S}_{1}=J=m a, \mathcal{M}_{2}=q-m a^{2}, \mathcal{S}_{3}\right.$, and $\left.\mathcal{M}_{4}\right)$. To see if it is true, one has to construct the Ernst potential for this metric. This potential is given by [5] 


$$
\mathcal{E}=f+i \Omega,
$$

where $f=V=V_{K} \mathrm{e}^{-2 \psi}$ and $\Omega$ is the twist scalar. To get this scalar, the following equation has to be solved

$$
\partial_{\alpha} \Omega=\varepsilon_{\alpha \beta \mu \nu} k^{\beta} \nabla^{\mu} k^{\nu}
$$

where $k^{\beta}$ is the Killing vector, $\nabla^{\mu}$ is the contravariant derivative and $\varepsilon_{\alpha \beta \mu \nu}=\sqrt{-g} \epsilon_{\alpha \beta \mu \nu}(g$ is determinant of the metric tensor). Let us take the Killing vector as in the Kerr metric $k^{\beta}=$ $(1,0,0,0)$. Then, the approximate solution of $(20)$ is

$$
\Omega=-2 \frac{J}{\rho^{2}} \cos \theta+\mathcal{H}
$$

where

$$
\mathcal{H}=\frac{S_{3}}{r^{4}} h_{61}+\frac{J q}{r^{5}} h_{62}+\frac{m S_{3}}{r^{5}} h_{63}+\frac{q S_{3}}{r^{7}} h_{64}+\frac{J M_{4}}{r^{7}} h_{65}+\frac{M_{4} S_{3}}{r^{9}} h_{66},
$$

with

$$
\begin{aligned}
h_{61} & =\frac{7}{12}\left(5 P_{2}-2\right) \cos \theta, \\
h_{62} & =4 P_{2} \cos \theta, \\
h_{63} & =\frac{1}{420}\left(490\left(5 P_{2}-2\right) \cos \theta-96 P_{4}\right), \\
h_{64} & =\frac{1}{6}\left(4 P_{2}-18 P_{4}-7\right) \cos \theta, \\
h_{65} & =4 P_{4} \cos \theta, \\
h_{66} & =\frac{1}{66}\left(-110 P_{2}+54 P_{4}-175 P_{6}\right) \cos \theta .
\end{aligned}
$$

Now, the Ernst function is given by

$$
\xi=\frac{1+\mathcal{E}}{1-\mathcal{E}}
$$

It is easy to show that this Ernst function and its inverse are solutions of the Ernst equation [5]

$$
\left(\xi \xi^{\star}-1\right) \nabla^{2} \xi=2 \xi^{\star}[\nabla \xi]^{2} .
$$

To calculate the relativistic multipole moments, it is better to employ the inverse function [5]. Moreover, it is custumary to employ the prolate spheroidal coordinates $(t, x, y, \phi)$. The transformation to these coordinates is achieved by means of

$$
\begin{aligned}
\sigma x & =r-M, \\
y & =\cos \theta,
\end{aligned}
$$

where $\sigma^{2}=M^{2}-a^{2}$.

The method to obtain the relativistic multipole moments is the following [6] 
1. use the inverse Ernst function $\xi^{-1}$ in prolate coordinates,

2. set $y=\cos \theta=1$ into $\xi^{-1}$,

3. change $\sigma x \rightarrow 1 / z$ into $\xi^{-1}$,

4. expand in Taylor series of $z$ the inverse Ernst function, and finally,

5. employ the Fodor-Hoenselaers-Perjés (FHP) formulae [6].

A REDUCE program that calculates the multipole moment was written with this recipe. The first ten complex moments $\mathcal{P}_{n}=\mathcal{M}_{n}+i \mathcal{S}_{n}$ are

$$
\begin{aligned}
\mathcal{P}_{0} & =\mathcal{M}_{0}=m \\
\mathcal{P}_{1} & =i \mathcal{S}_{1}=i J=i m a \\
\mathcal{P}_{2} & =\mathcal{M}_{2}=q-m a^{2} \\
\mathcal{P}_{3} & =i \mathcal{S}_{3}=-i\left(m a^{3}+\frac{7}{8} S_{3}\right) \\
\mathcal{P}_{4} & =\mathcal{M}_{4}+i \mathcal{S}_{4}=m a^{4}+M_{4}+i\left(-2 J q+\frac{4}{35} m S_{3}\right) \\
\mathcal{P}_{5} & =\mathcal{M}_{5}+i \mathcal{S}_{5}=\frac{7}{4} J S_{3}-4 m M_{4}+i m a^{5} \\
\mathcal{P}_{6} & =\mathcal{M}_{6}+i \mathcal{S}_{6}=-m a^{6}+i\left(\frac{7}{4} q S_{3}-2 J M_{4}\right) \\
\mathcal{P}_{7} & =\mathcal{M}_{7}+i \mathcal{S}_{7}=\left(\frac{1}{5} q M_{4}+\frac{16}{429} S_{3}^{2}\right)-i m a^{7}, \\
\mathcal{P}_{8} & =\mathcal{M}_{8}+i \mathcal{S}_{8}=m a^{8}+i \frac{7}{4} M_{4} S_{3}, \\
\mathcal{P}_{9} & =i \mathcal{S}_{9}=i m a^{9} \\
\mathcal{P}_{10} & =\mathcal{M}_{10}=-m a^{10} .
\end{aligned}
$$

The real parts are the massive multipoles, $\mathcal{M}_{i}$ and the imaginary parts are the spin multipoles, $\mathcal{S}_{i}$. If one eliminates mixed terms and $S_{3}^{2} \sim 0$ in (26) the multipole structure becomes simpler.

For neutron stars, the form of the first five multipole moments are [17, 21, 16]

$$
\begin{aligned}
\mathcal{M}_{0} & =m, \\
\mathcal{S}_{1} & =S=J=m a, \\
\mathcal{M}_{2} & =-\alpha m a^{2}, \\
\mathcal{S}_{3} & =-\beta m a^{3}, \\
\mathcal{M}_{4} & =\gamma m a^{4},
\end{aligned}
$$

where $\alpha, \beta$, and $\gamma$ are parameters.

It is easy to see that if one sets

$$
\begin{aligned}
q & =(1-\alpha) m a^{2}, \\
S_{3} & =-\frac{7}{8}(\beta+1) m a^{3}, \\
M_{4} & =(\gamma-1) m a^{4},
\end{aligned}
$$

a similar multipole structure is obtained from (26). 
Now, let us determine the multipole structure of the new HT spacetime. The twist scalar for this HT metric is

$$
\begin{aligned}
\Omega & =\left[-2 J u^{2}+\frac{7}{12} S_{3}\left(5 P_{2}-2\right) u^{4}+\frac{1}{6}\left(-24 J q P_{2}+7 M S_{3}\left(5 P_{2}-2\right)\right) u^{5}\right. \\
& \left.+\frac{1}{6}\left(-24 J M_{4} P_{4}+q S_{3}\left(-4 P_{2}+18 P_{4}+7\right)\right) u^{7}+\frac{1}{66} M_{4} S_{3}\left(110 P_{2}-54 P_{4}+175 P_{6}\right) u^{9}\right] \cos \theta .
\end{aligned}
$$

After using (15) and (29) to construct the Ernst functions, we find that the relativistic multipole moments for this HT metric are

$$
\begin{aligned}
\mathcal{M}_{0} & =M, \\
\mathcal{S}_{1} & =J, \\
\mathcal{M}_{2} & =-q, \\
\mathcal{S}_{3} & =-\frac{7}{8} S_{3}, \\
\mathcal{M}_{4} & =M_{4} .
\end{aligned}
$$

Obviously, from this multipole structure, it is possible to calculate the multipole moments of a neutron star, as well.

\section{Comparison with the Pappas Metric}

Pappas found an approximate solution of the EFE by means of the Ernst method [17]. This spacetime has 5 parameters $M, M_{2}, M_{4}, S_{1}=J, S_{3}$, which, by construction, represent the relativistic multipole moments. The metric is given in cylindrical Weyl-Papapetrou coordinates by

$$
d s^{2}=-f(d t-\omega d \phi)^{2}+\frac{1}{f}\left[\mathrm{e}^{2 \gamma}\left(d \rho^{2}+d z^{2}\right)+\rho^{2} d \phi^{2}\right]
$$

where

$$
\begin{aligned}
f & =1-2 \frac{M}{\eta}+2 \frac{M^{2}}{\eta^{2}}+\frac{1}{\eta^{5}}\left[\left(M_{2}-M^{3}\right) \rho^{2}-2\left(M^{3}+M_{2}\right) z^{2}\right] \\
& +\frac{1}{\eta^{6}}\left[2 z^{2}\left(M^{4}-J^{2}+2 M M_{2}\right)-2 M M_{2} \rho^{2}\right]+\frac{A}{28 \eta^{9}}+\frac{B}{14 \eta^{10}} \\
\omega & =-2 \frac{J}{\eta^{3}} \rho^{2}-2 \frac{M J}{\eta^{4}} \rho^{2}+\frac{F}{\eta^{7}}+\frac{H}{2 \eta^{8}}+\frac{G}{4 \eta^{11}}, \\
\gamma & =\frac{1}{4 \eta^{8}} \rho^{2}\left[J^{2}\left(\rho^{2}-8 z^{2}\right)+M\left(M^{3}+3 M_{2}\right)\left(\rho^{2}-4 z^{2}\right)\right]-\frac{M^{2}}{2 \eta^{4}} \rho^{2},
\end{aligned}
$$

with

$$
\begin{aligned}
\eta & =\sqrt{\rho^{2}+z^{2}} \\
A & =8 \rho^{2} z^{2}\left(24 M J^{2}+17 M^{2} M_{2}+21 M_{4}\right)+\rho^{4}\left(7 M^{5}-10 M J^{2}+32 M^{2} M_{2}-21 M_{4}\right) \\
& +8 z^{4}\left(-7 M^{5}+20 M J^{2}-22 M_{2} M^{2}-7 M_{4}\right),
\end{aligned}
$$




$$
\begin{aligned}
B & =\rho^{4}\left(10 M^{2} J^{2}+10 M^{3} M_{2}+21 M M_{4}+7 M_{2}^{2}\right) \\
& +4 z^{4}\left(7 M^{6}-40 M^{2} J^{2}-14 J S_{3}+30 M^{3} M_{2}+14 M M_{4}+7 M_{2}^{2}\right) \\
& -4 \rho^{2} z^{2}\left(7 M^{6}+27 J^{2} M^{2}-21 J S_{3}+48 M^{3} M_{2}+42 M M_{4}+7 M_{2}^{2}\right), \\
F & =\rho^{4}\left(S_{3}-M^{2} J\right)-4 \rho^{2} z^{2}\left(M^{2} J+S_{3}\right), \\
G & =\rho^{2}\left(-J^{3}\left(\rho^{4}+8 z^{4}-12 \rho^{2} z^{2}\right)+M J\left(\left(M^{3}+2 M_{2}\right) \rho^{4}\right.\right. \\
& \left.\left.-8\left(3 M^{3}+2 M_{2}\right) z^{4}+4\left(M^{3}+10 M_{2}\right) \rho^{2} z^{2}\right)+M^{2} S_{3}\left(3 \rho^{4}-40 z^{4}+12 \rho^{2} z^{2}\right)\right), \\
H & =4 \rho^{2} z^{2}\left(J\left(M_{2}-2 M^{3}\right)-3 M S_{3}\right)+\rho^{4}\left(J M_{2}+3 M S_{3}\right) .
\end{aligned}
$$

To see which form has this metric in spherical-like coordinates, we use the Kerr mapping (2) with $a=0$. Then, the function $\eta^{2}$ is

$$
\eta^{2}=\Delta+\left(M^{2}-a^{2}\right) \cos ^{2} \theta=r(r-2 M)+M^{2} \cos ^{2} \theta .
$$

Substituting (34) in the metric functions (32) and expanding in Taylor series up to $\mathcal{O}\left(r^{-6}\right)$, the metric potentials take the form

$$
\begin{aligned}
& V=f \\
& =1-2 M u-2 M_{2} P_{2} u^{3}-\frac{2}{3}\left[J^{2}\left(2 P_{2}+1\right)+3 M M_{2} P_{2}\right] u^{4} \\
& +\frac{1}{63}\left[-3 M J^{2}\left(14 P_{2}^{2}+20 P_{2}+14\right)-M M_{2}\left(70 P_{2}^{2}+88 P_{2}-14\right)\right. \\
& \left.+7 M_{4}\left(-35 P_{2}^{2}+10 P_{2}+7\right)\right] u^{5}, \\
& W=f \omega \\
& =\left[-2 J u-\frac{2}{3} S_{3} u^{3}\left(5 P_{2}+1\right)+\frac{1}{3}\left[3 J M_{2}-5 M S_{3}\right] u^{4}\left(5 P_{2}+1\right)\right. \\
& +\frac{1}{3}\left[J^{3}\left(-7 P_{2}^{2}+8 P_{2}+5\right)+6 M J M_{2}\left(-3 P_{2}^{2}+7 P_{2}+2\right)\right. \\
& \left.\left.+\quad M^{2} S_{3}\left(7 P_{2}^{2}-50 P_{2}-11\right)\right] u^{5}\right] \sin ^{2} \theta, \\
& X=\frac{1}{f \Delta}\left[(r-M)^{2} \sin ^{2} \theta+\Delta \cos ^{2} \theta\right] \mathrm{e}^{2 \gamma} \\
& =1+2 M u+4 M^{2} u^{2}+2\left(4 M^{3}+M_{2} P_{2}\right) u^{3} \\
& +\frac{2}{3}\left[24 M^{4}+3 J^{2} P_{2}^{2}+M M_{2}\left(5 P_{2}^{2}+11 P_{2}-1\right)\right] u^{4} \\
& +\frac{1}{63}\left[2016 M^{5}+3 M J^{2}\left(266 P_{2}^{2}-36 P_{2}-14\right)+M^{2} M_{2}\left(1330 P_{2}^{2}+1096 P_{2}-266\right)\right. \\
& \left.+\quad 7 M_{4}\left(35 P_{2}^{2}-10 P_{2}-7\right)\right] u^{5}, \\
& Y=\frac{1}{f}\left[(r-M)^{2} \sin ^{2} \theta+\Delta \cos ^{2} \theta\right] \mathrm{e}^{2 \gamma} \\
& =r^{2}\left[1+2 M_{2} u^{3} P_{2}+\frac{2}{3}\left[3 J^{2} P_{2}^{2}+M M_{2}\left(5 P_{2}^{2}+5 P_{2}-1\right)\right] u^{4}\right. \\
& +\frac{1}{63}\left[3 M J^{2}\left(182 P_{2}^{2}-36 P_{2}-14\right)+M^{2} M_{2}\left(910 P_{2}^{2}+172 P_{2}-182\right)\right. \\
& \left.\left.+\quad 7 M_{4}\left(35 P_{2}^{2}-10 P_{2}-7\right)\right] u^{5}\right] \text {, } \\
& Z=\frac{\rho^{2}}{f}-f \omega^{2}
\end{aligned}
$$




$$
\begin{aligned}
& =r^{2} \sin ^{2} \theta\left[1+2 M_{2} P_{2} u^{3}+2\left(J^{2}\left(2 P_{2}-1\right)+3 M M_{2} P_{2}\right) u^{4}\right. \\
& +\frac{1}{63}\left[3 M J^{2}\left(14 P_{2}^{2}+188 P_{2}-70\right)+M^{2} M_{2}\left(70 P_{2}^{2}+844 P_{2}-14\right)\right. \\
& \left.\left.-\quad 7 M_{4}\left(35 P_{2}^{2}-10 P_{2}-7\right)\right] u^{5}\right]
\end{aligned}
$$

where $u=1 / r$. By means of a REDUCE program, we checked that the metric potentials fulfill the EFE. According to Pappas the multipole structure of his spacetime is $\mathcal{M}_{2 n}=M, M_{2}, M_{4}$ for

$n=0,1,2$ and $\mathcal{S}_{2 n+1}=S_{1}, S_{3}$ for $n=0,1$. The twist potential for the expanded Pappas spacetime is

$$
\Omega=-\frac{1}{3}\left[6 J u^{2}+S_{3} u^{4}\left(10 P_{2}-4\right)+u^{5}\left(12 J M_{2} P_{2}+M S_{3}\left(20 P_{2}-8\right)\right)\right] \cos \theta .
$$

Using (35) and (36) in our program, the multipole structure is as expected

$$
\begin{aligned}
\mathcal{M}_{0} & =M, \\
\mathcal{S}_{1} & =J, \\
\mathcal{M}_{2} & =M_{2}, \\
\mathcal{S}_{3} & =S_{3}, \\
\mathcal{M}_{4} & =M_{4} .
\end{aligned}
$$

Then, our metric (3) have not the same multipole structure, therefore they are not isometric. The multipole structures of the new HT and the Pappas metrics are similar. Setting $q=-M_{2}$ and rescaling $S_{3}$ in (30) both metrics become isometric. From (35), the Pappas metric contains the post-linear versions of the Schwarzschild, Erez-Rosen (up to $M M_{2}$ ) and the Lense-Thirring metrics. Our metric contains the Kerr metric and the post-linear version of the Erez-Rosen metric (up to second order in $q$ ).

\section{Conclusions}

We have found an approximate solution of the EFE by means of perturbing the Kerr metric. This approximate solution has five parameters, mass, angular momentum, mass quadrupole, spin octupole and mass hexadecapole. The mass quadrupole, spin octupole and mass hexadecapole were included perturbatively. It is valid up to the third order in these parameters.

By finding the twist scalar, we found the multipole structure employing the FHP formalism. It is possible to choose the multipole parameters, so that the first five multipole moments of a neutron star are similar. The simple form of our spacetime does it easy to implement computationaly. Including more relativistic multipole moments to our metric is easy using the procedure described here. Our metric presents an advantage over the Pappas metric, because it does not contain the Kerr spacetime as a limiting case.

Through a transformation we guessed an improved HT metric at first order in the spin octupole and mass hexadecapole. Solving the EFE, a new version of the HT, including mixed and cuadratic terms was also found. This is important, because the original HT can be matched with interior solutions and is used to validate spacetimes. The twist scalars and the multipole structures were also found for this improved HT and the Pappas metric. A comparison reveals that this HT metric is isometric with the Pappas metric after doing a transformation. 
Our spacetime has potentialy many applications. It could be used to infer the properties of the structure of a neutron star from astrophysical observations. Another task is to find the ISCO as a function of the mass, mass quadrupole, mass hexadecapole, spin and spin octupole, as an extention of [4]. An interesting future work is to find an interior solution for our spacetime.

\section{References}

[1] E. Berti et al., Testing general relativity with present and future astrophysical observations Classical and Quantum Gravity, 32(24), 243001, 2015. https://dx.doi.org/10.1088/0264$9381 / 32 / 24 / 243001$

[2] E. Berti, F. Frances, A.Maniopoulou and M: Bruni, Rotating neutrons stars: An invariant comparison of approximate and numerical spacetime models, Monthly Notices of the Royal Astronomical Society, 358, 923-938, 2005. https://doi.org/10.1111/j.1365-2966.2005.08812.x

[3] M. Camenzind, Compact Objects in Astrophysics, Springer, Berlin, 2007.

[4] F. Chaverri-Miranda, F. Frutos-Alfaro, P. Gómez-Ovarez, A. Oliva-Mercado, Innermost stable circular orbits of a Kerr-like Metric with Quadrupole, https://arxiv.org/pdf/1707.08663.pdf, 2017.

[5] F. J. Ernst, New Formulation of the Axially Symmetric Gravitational Field Problem, Physical Review, 167(5), 1175-1177, 1968. http://dx.doi.org/10.1103/PhysRev.167.1175

[6] G. Fodor, C. Hoenselaers, and Z. Perjés, Multipole moments of axisymmetric systems in relativity, Journal of Mathematical Physics, 30, 2252-2257, 1989. http://dx.doi.org/10.1063/1.528551

[7] F. Frutos-Alfaro, E. Retana-Montenegro, I. Cordero-García, J. Bonatti-González, Metric of a Slow Rotating Body with Quadrupole Moment from the Erez-Rosen Metric, International Journal of Astronomy and Astrophysics, 3(4), 431-437, 2013.

https://dx.doi.org/10.4236/ijaa.2013.34051

[8] F. Frutos-Alfaro, P. Montero-Camacho, M. Araya-Arguedas, J. Bonatti-González, Approximate Metric for a Rotating Deformed Mass, International Journal of Astronomy and Astrophysics, 5(1), 1-10, 2015.

https://dx.doi.org/10.15517/10.4236/ijaa.2015.51001

[9] F. Frutos-Alfaro, M. Soffel, On the Post-linear Quadrupole-Quadrupole Metric, Revista de Matemática: Teoría y Aplicaciones, 24(2), 239-255, 2017.

https://dx.doi.org/10.15517/rmta.v24i2.29856

[10] F. Frutos-Alfaro, Approximate Kerr-like Metric with Quadrupole, International Journal of Astronomy and Astrophysics, 6, 334-345, 2016. https://dx.doi.org/10.4236/ijaa.2016.63028

[11] J. B. Hartle and K. S. Thorne, Slowly Rotating Relativistic Stars. II Models for Neutron Stars and Supermassive Stars, The Astrophysical Journal, 153, 807-834, 1968.

https://dx.doi.org/10.1086/149707

[12] J. M. Lattimer and M. Prakash, The Physics of Neutron Stars, Science, 304, 536-542, 2004. https://dx.doi.org/10.1126/science.1090720

[13] T. Lewis, Some Special Solutions of the Equations of Axially Symmetric Gravitational Fields, Proceedings of the Royal Society London A, 176-192, 1932. https://dx.doi.org/10.1098/rspa.1932.0073

[14] V. S. Manko and I. D. Novikov, Generalizations of the Kerr and Kerr-Newman Metrics Possessing an Arbitrary Set of Mass-Multipole Moments, Classical and Quantum Gravity, 9, 2477-2487, 1992. https://dx.doi.org/10.1088/0264-9381/9/11/013 
[15] L. A. Pachón, J. A. Rueda and J. D. Sanabria-Gómez, Realistic exact solution for the exterior field of a rotating neutron star, Physical Review D, 73(10), id. 104038, 2006.

https://dx.doi.org/10.1103/PhysRevD.73.104038

[16] G. Pappas and T. A. Apostolatos, Multipole moments of numerical spacetimes, https://arxiv.org/pdf/1211.6299.pdf, 2012.

[17] G. Pappas, An accurate metric for the spacetime around neutron stars, Monthly Notices Royal Astronomical Society, 466(4), 4381-4394, 2017. https://dx.doi.org/10.1093/mnras/stx019

[18] H. Quevedo and B. Mashhoon, Generalization of Kerr spacetime, Physical Review D, 43(12), 3902-3906, 1991. https://dx.doi.org/10.1103/PhysRevD.43.3902

[19] F. D. Ryan, Spinning boson stars with large self-interaction, Physical Review D, 55(10), 60816091, 1997. https://doi.org/10.1103/PhysRevD.55.6081

[20] N. Stergioulas, Rotating Stars in Relativity, Living Reviews in Relativity, 2003. https://dx.doi.org/10.12942/lrr-2003-3

[21] Yagi K., Kyutoku K., Pappas G., Yunes N., Apostolatos T. A., Effective no-hair relations for neutron stars and quark stars: Relativistic results, Physical Review D, 89, 124013, 2014. https://doi.org/10.1103/PhysRevD.89.124013 